\title{
Experiencia en la intervención con personas mayores en situación de dependencia en la Fundación Matia
}

\author{
Experience in interventions with elderly dependent \\ persons at the Matia Foundation
}

Francisco Javier LETURIA ARRAZOLA*

\section{RESUMEN}

Fundación Matia es una institución privada de carácter social cuya labor está dirigida fundamentalmente a preservar y mejorar la calidad de vida de las personas mayores y personas con discapacidad.

En los últimos años, dentro de la Fundación se está desarrollando un gran proceso de cambio organizacional basado en el desarrollo del modelo psicosocial y sociosanitario, la especialización y personalización en la atención, la generación de nuevos recursos especializados, el desarrollo de tres áreas de intervención fundamentales como son el área sociosanitaria, el área psicosocial y el área de integración y participación sociocomunitaria. Todo ello, desarrollando a la vez los más avanzados sistemas de gestión.

El modelo de intervención psicosocial se basa en el carácter interdisciplinar y de complementariedad entre las diferentes disciplinas implicadas, con la complementación de las aportaciones que la gerontología conductual ofrece para el diseño ambiental, la atención, los tratamientos, etc. con una población que presenta una alta prevalencia de situaciones de dependencia, patología neurológica y psiquiátrica, déficits sensoriales, etc.

Uno de los principales objetivos que nos planteamos a partir de este modelo es ofrecer a través de un equipo de profesionales competentes, organizados interdisciplinarmente, una atención profesional, complementaria a la relación familiar y social, de alta calidad, a la vez que muy profesional, muy cercana, personalizada y humana, incorporando al voluntariado y considerando a la familia como el eje central de la intervención con el usuario y residente.

En cuanto al modelo de atención residencial, tenemos como objetivo la ejecución, siguiendo la planificación estratégica de las actividades que se llevan a cabo en los centros y unidades gerontológicas y residenciales para lograr la mejor calidad de vida de las

* Director Técnico de Servicios Sociales y de Gestión de Conocimiento. Fundación Matia. INGEMA. Instituto Gerontológico Matia. 
personas mayores atendidas y sus familias, así como garantizar y promover sus derechos consiguiendo satisfacer al máximo sus expectativas, con la máxima eficacia y eficiencia.

Dentro de este modelo de atención destacamos como aspectos primordiales, la influencia del entorno en la calidad de vida y el carácter integral de la intervención.

A partir de la implantación del nuevo modelo de atención e intervención psicosocial de manera integrada a los modelos de gestión más avanzado y a un enfoque abierto a la comunidad y orientado al cliente, el área psicosocial de Matia Fundazioa ha sufrido una importante evolución en los últimos años.

\title{
PALABRAS CLAVE
}

Intervención psicosocial, Gerontología, Dependencia, Personas mayores, Calidad de vida, Atención residencial, Atención sociosanitaria.

\begin{abstract}
The Matia Foundation is a private social institution that focuses its activity on safeguarding and improving the quality of life of the elderly and the disabled.

In recent years, an extensive process of organisational change has been carried out within the Foundation, based on the development of the psychosocial and social-health model, the specialisation and personalisation of the care, the generation of new specialised resources and the development of three fundamental areas of intervention. These three areas correspond to the Social Health Area, the Psychosocial Area and the Social/Community Integration and Participation Area. All of this has been carried out in conjunction with the development of the most advanced management systems.
\end{abstract}

The Psychosocial Intervention Model works in an interdisciplinary and complementary way between the different disciplines involved, in addition to the contributions that behavioural gerontology offers for the ambient design, care and treatments, etc. with a population that displays a high prevalence of dependent situations, neurological and psychiatric pathology, sensorial deficiencies etc.

One of the main objectives we aim to meet via this model is to complement the family and social relationship with a competent team of professionals, organised according to their disciplines, offering high quality, highly professional care, which is friendly, personalised and humane, by incorporating voluntary staff and taking into consideration the family as the central point of the intervention with the user and resident.

As regards the Residential Care Model, we have aim to ensure execution by following the strategic planning of the activities carried out in the gerontology and residential centres and units in order to achieve a better quality of life for the elderly people in our care along with their families, in addition to guaranteeing and promoting their rights in order to fully satisfy their expectations with the maximum effectiveness and efficiency.

Within this care model we can highlight the following fundamental aspects: the influence of the surroundings on the quality of life and the integral nature of the intervention.

Through the integrated introduction of the new more advanced Psychosocial Care and Intervention Model to the most advanced management models and to a client-based focus that is open to the community, the Psychosocial Area of the Matia Foundation has experienced an important evolution in recent years.

\section{KEY WORDS}

Psychosocial Intervention, Gerontology, Dependency, Elderly People, Quality of Life, Residential Care, Social Health Care. 


\section{INTRODUCCIÓN}

Las personas mayores constituyen un colectivo que, aunque heterogéneo, presenta cada vez más claramente unas características fundamentales que se agrupan en torno a tres situaciones diferenciadas: un gran número de personas mayores jóvenes se encuentran cada vez mejor y pueden ser entendidas bajo el paradigma del envejecimiento exitoso y la intervención psicosocial utilizando las estrategias de optimización selectiva con compensación (Baltes y Baltes, 1990), otro gran grupo que se encuentra alrededor de las situaciones de dependencia, es decir, necesitan el apoyo de terceras personas para desenvolverse en la vida cotidiana, así como un tercero en el que se agrupan aquellas que presentan un frágil equilibrio en cuanto a salud y autonomía, sufren polimorbilidad, etc. (Baltes y Wahl, 1990) y tienen el riesgo de convertirse en dependientes.

En el primer caso consideraremos aspectos como los limites del funcionamiento en la edad avanzada y las condiciones que permiten su mantenimiento óptimo, el potencial de aprendizaje y la capacidad entrenable y/o prevenible, desde un enfoque multicriterial que considera la vitalidad, resistencia, flexibilidad adaptativa, autonomía, control, integridad, buen ajuste persona-ambiente la variabilidad interindividual y la plasticidad intraindividual y capacidad de aprendizaje de las personas mayores. En el caso de la dependencia, si bien la mayoría de trabajos y enfoques se centran en la dependencia física y la necesidad de apoyos para las actividades de la vida diaria con un enfoque unidimensional, parece necesario conceptualizarlo como un fenómeno multicausal y con un componente psicológico y contextual muy importante sobre el que se debe intervenir (Montorio, 1998, Montorio y Losada, 2004).
La gerontologia conductual, entendida como disciplina que estudia la aplicación de los principios de la psicologia cientifica a la solución de problemas de la vejez (Montorio, 1995), presenta un interés preferente no sólo en la intervención individual y grupal, sino también en la planificación, organización, en la implementación de planes de intervención y en la evaluación de programas y servicios(Yanguas y Leturia, 1995a y 1995b), o de manera más específica en la optimización de los procesos de adaptación al entorno y las nuevas condiciones que surgen con la edad, la prevención de problemas de mayor prevalencia en las diferentes áreas vitales del sujeto y la intervención en esas áreas, llegando hasta el manejo de trastornos de comportamiento de alta prevalencia en los centros de atención (ya sea esta de tipo residencial, diurna, etc). En cualquier caso, el supuesto del que partimos es que la dependencia tiene un componente psicosocial que se puede prevenir y sobre el cual se debe intervenir (Montorio, 1998).

\section{CONTEXTO DE APLICACIÓN EN FUNDACIÓN MATIA}

Fundación Matia es una institución privada de carácter social creada en 1888, que actualmente gestiona un continuo de servicios y centros que abarcan 6 centros gerontológicos con 600 plazas y 4 centros de día con 123 plazas , un centro de atención residencial especializada para personas con discapacidad con 56 plazas orientadas a casos de daño cerebral, lesiones medulares y problemas neurológicos fundamentalmente, un hospital de media larga estancia con servicios de rehabilitación, geriatría y convalecencia con 88 plazas, y unidad de cuidados paliativos de 15 plazas, un servicio de rehabilitación que hace 5626 diagnósticos, 9207 revisiones y 188083 
tratamientos anuales y 12474 tratamientos de logopedia.

Desde su actual definición de empresa y su cultura empresarial está realizando un proceso de cambio organizacional importante en los últimos años tal y como se refleja en los planes estratégicos $1998-2000$ y 2001-2003 y en el actual 2004-2007, así como en los resultados expuestos en las memorias anuales.

Este cambio puede resumirse en:

1. Desarrollo del modelo psicosocial y sociosanitario .

2. Especialización y personalización en la atención.

3. Generación de nuevos recursos especializados como el SEOG Servicio de Evaluación y Orientación Gerontológica ( 5285 valoraciones en el año 2005) y la UMA Unidad de Memoria y Alzheimer (362 valoraciones /año 2005)

4. Desarrollo de tres áreas de intervención fundamentales:

- Área sociosanitaria.

- Área psicosocial.

- Área de integración y participación sociocomunitaria.

5. Desarrollo de la gestión del conocimiento tanto en la gestión diaria en los procesos y centros como con la creación de INGEMA Instituto Gerontológico Matia ( reconocida como agente científico tecnológico de la Red Vasca de Ciencia y Tecnología por el Gobierno Vasco)

6. Desarrollo de los más avanzados sistemas de gestión: gestión de las personas por competencias, gestión de la calidad a través del aseguramiento y de los modelos de mejora continua y excelencia.

7. Creación de la nueva empresa Gerozerlan

Desde el año 2001 todos los Centros Gerontológicos y de Día al igual que el resto de Centros y Servicios de la Fundación están certificados según ISO 9000, desde 2003 según ISO 9001: 2000, ISO 14001 y OHSAS 18001. Desde 2004 están reconocidos con la $Q$ de plata según modelo EFQM. En los últimos años se han logrado los Premios Infanta Cristina, Premios Caja Madrid a la Investigación y Dolores Ibarruri del Gobierno vasco a los Servicios Sociales etc. Desde 2006 están reconocidos con la QORO según modelo EFQM.

\section{Quiénes somos}

En Matia Fundazioa trabajamos en total 587 personas, en los propios centros y apoyando a estos desde los servicios centrales de la Fundación. Parte somos trabajadores de la fundación (422) y contamos con la colaboración de diferentes empresas que complementan nuestra actuación como son: SUMAN, EULEN, LAHAR, o las empresas del GRUPO GUREAK. Contamos en nuestros equipos con un importante número de personas discapacitadas 17 en plantilla y 247 subcontratadas, que están perfectamente integradas y desarrollan su actividad de la manera más eficiente.

Todos intentamos trabajar con la metodologia interdisciplinar y participativa porque creemos que "la manera de conseguir nuestros objetivos es lograr equipos profesionales competentes que con metodología interdisciplinar y participativa consiguen ofrecer la máxima 
calidad de atención tal y como se define en la Visión de empresa, y además valoremos mucho la satisfacción de las personas que compartimos el proyecto de Matia Fundazioa, así como la sostenibilidad del mismo.

Los equipos están compuestos entre otros por 57 DUEs, 12 DTS, 7 psicologos, 8 monitores grupales , 2 terapeutas ocupacionles, 16 fisioterapeutas, 23 medicos, 2 farmaceuticos. Y este trabajo lo realizamos con un modelo integrador de las personas trabajadoras, las familias, los residentes y los voluntarios.

\section{El modelo de Intervención Psicosocial}

Dada la diversidad de situaciones y entornos en los que se debe intervenir es fundamental consensuar un modelo de intervención psicosocial que permita una adecuada fundamentación, estructuración y definición de los planes y programas de atención e intervención y permita dar una lógica integral a cada uno de los programas.

Este modelo y plan de intervención se basa en el carácter interdisciplinar (Leturia, Leturia y Yanguas, 1998) y de complementariedad entre las diferentes disciplinas implicadas. En los equipos de trabajo el modelo psicosocial se complementa con las aportaciones que la gerontologia conductual ofrece para el diseño ambiental, la atención, los tratamientos etc. con una población que presenta una alta prevalencia de situaciones de dependencia, patología neurológica y psiquiátrica, déficits sensoriales, etc.

\section{Modelo de Influencia ( figura 1)}

Los objetivos principales de la atención y cuidado en el Centro Gerontológico son:
1. Ofrecer una de las alternativas convivenciales y de atención acordes con las necesidades de los usuarios y sus familias.

2. Conocer a través de la valoración integral realizada por el equipo interdisciplinar, las capacidades y necesidades físicas, psiquicas , sociales y funcionales de las personas atendidas y usuarias del centro.

3. Ofrecer los cuidados y atención personalizada que los residentes y usuarios necesitan para desenvolverse en la vida diaria, a partir de un plan general de intervención y de planes personalizados, para lograr un adecuado nivel de satisfacción y bienestar y por tanto de calidad de vida.

4. Mantener las capacidades y competencia funcional y social, las relaciones sociales y familiares, logrando que el centro sea un espacio de promoción y desarrollo personal y grupal.

5. Mantener y promover la relación del centro y de los residentes y usuarios con la comunidad más cercana, con el fin de impulsar los principios de normalización e integración establecida en la legislación vigente.

6. Prevenir el deterioro físico, psíquico y/o funcional o la disminución de la interacción social, propiciando unos adecuados tratamientos, programas de estimulación, actividades sociales, etc.

7. Ofrecer a través de un equipo de profesionales competentes, organizados interdisciplinarmente, una atención profesional, complementaria a la relación familiar 
Figura 1. Modelo de influencia

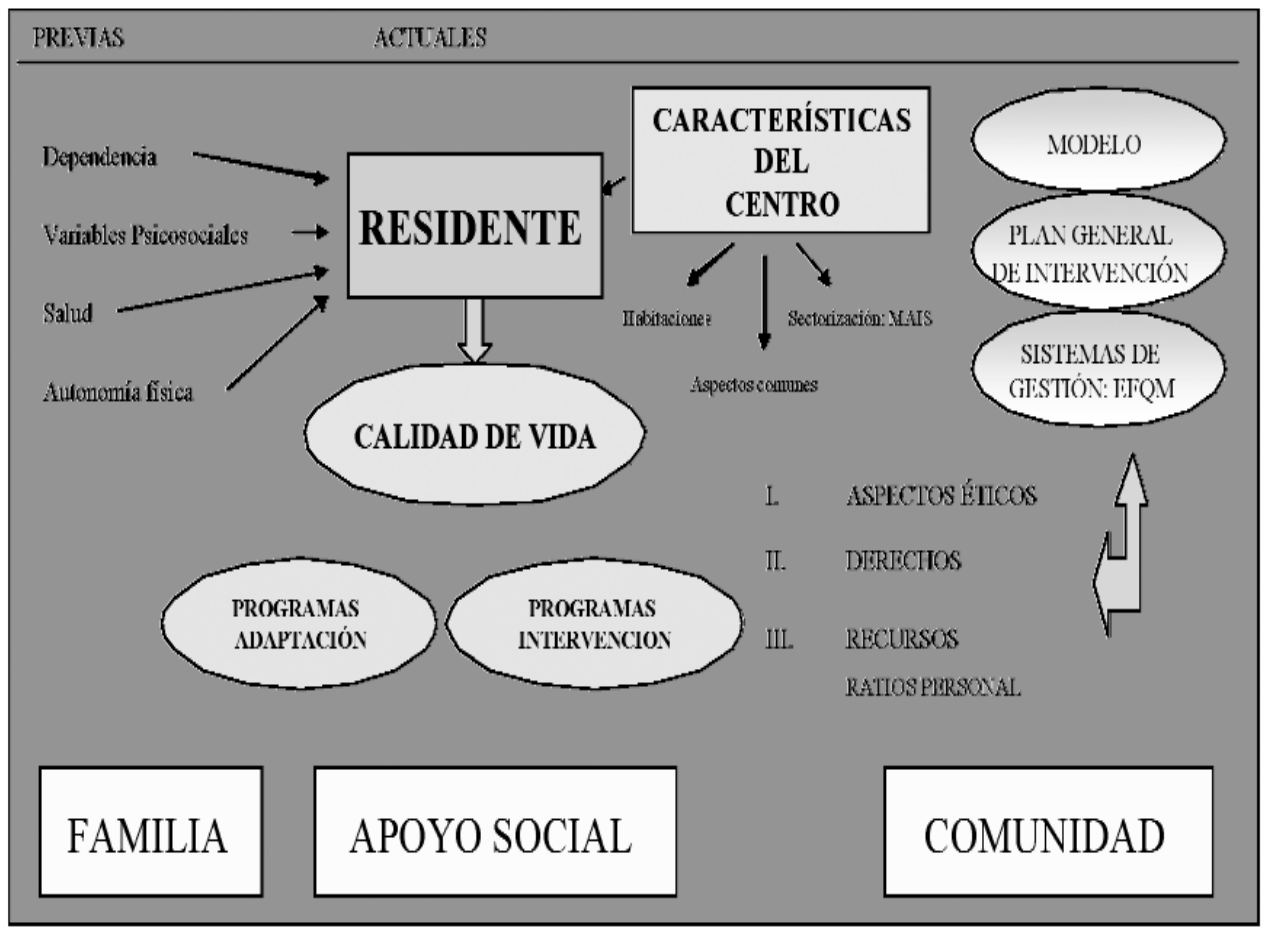

y social, de alta calidad, a la vez que muy profesional, muy cercana, personalizada y humana, incorporando al voluntariado y considerando a la familia como el eje central de la intervención con el usuario y residente.

8. Promover al máximo, además de respetar, los derechos individuales y colectivos de las personas atendidas.

9. Promover una imagen positiva de la vejez y de los colectivos atendidos por su discapacidad o problemática, así como del cuidado a estas personas y de los centros socioasistenciales.
10. Promover el mantenimiento de las personas mayores atendidas en el Centro de Día en su domicilio y entorno familiar trabajado conjuntamente para hacerlo con la mejor calidad de vida para todos.

\section{ESTRATEGIAS:}

Para lograr estos objetivos nos planteamos las siguientes estrategias:

1. Estimulación. Se intentará establecer una estimulación adecuada para las personas atendidas considerando sus características psicológicas especialmente, así como 
sus capacidades psicofísicas y sociales, que fomente la interacción, la orientación y confort. A nivel ambiental se realizará a través de la decoración, a través de plantas, animales, música o radio etc. y a través de la comunicación en el trato y en cada una de las relaciones de ayuda. Se procurará que tengan una disposición activa y lo más autónoma posible según sus capacidades.

2. Orientación. Se procurará incluir información de orientación temporal y espacial de manera continua en los contactos y comunicaciones. Se pondrán los carteles oportunos. Se realizarán los grupos correspondientes para su fomento. Se establecen dispositivos para lograr una estimulación ambiental de carácter orientador.

3. Apoyo psicoafectivo. Por parte de todo el personal que trabaja en cada módulo de atención integral (MAI) se debe potenciar y ofrecer este apoyo, tendente a lograr un nivel de satisfacción y adaptación adecuado a sus circunstancias, aumentando esta necesidad a medida que el nivel de capacidades es menor, la agitación y la dependencia es mayor, cuando hay enfermedad y en los últimos momentos de la vida.

4. Promoción de la Autonomia física y salud. Además del control diario por parte del personal sanitario, y la complementación de cuidados personales, se desarrollará el programa de psicomotricidad y actividad física, así como el acompañamiento en paseos y movilización pasiva en los casos prescritos.

\section{Promoción de la Autonomia fun-}

cional. Se buscará desarrollar y mantener en lo posible la autonomía funcional para el desenvolvimiento en las actividades de la vida diaria. Se implementará un Programa de AVD, con una persona responsable del mismo y el personal cuidador que deberá seguir esas pautas.

\section{Promoción del Apoyo social y la} Socialización. Se fomentará en primer lugar que el personal se convierta en reforzante de comportamientos sociales, reforzando socialmente al residente, así como que entre los propios residentes se provean de este tipo de refuerzo.

7. Promoción del Apoyo familiar. Dentro del epígrafe anterior este es uno de los temas a desarrollar y potenciar. Se trabajará con las familias de manera individual y colectiva para potenciar su participación en las actividades y vida del módulo, a través de visitas, acompañamientos, colaboración en la decoración de los espacios personales , formación, participación en la Comisión, Cuestionarios de satisfacción, etc.

8. Contención en los casos de agitación y problemas de comportamiento. Uno de los objetivos importantes de la atención a personas con deterioro cognitivo es reducir los problemas de comportamiento y agitación que pueden presentarse en estas personas. Los medios a utilizar son los programas, la comunicación y relación tranquilizadora, las puertas de entrada, la música, el que dispongan de un espacio para desenvolverse y un espacio específico en caso de necesidad de contención. 
9. Personalización. Todos estos objetivos deben desarrollarse junto al de personalización, a través de planes individualizados, personalización de espacios y habitaciones etc. Se potenciará la visión de la persona en su conjunto y no solo desde el punto de vista del diagnóstico, la enfermedad o el déficit.

10. Normalización e integración. Junto a la personalización se potenciará la visión normalizadora e integradora en la atención, a través de que realicen actividades normales de cualquier hogar, reducir el aspecto hospitalario con una decoración y ambientación lo más parecida posible a la del hogar, realizar salidas etc.

11. Intimidad, autonomia, derechos y competencia en las decisiones: El respeto a la intimidad de los usuarios, el respeto a sus derechos, debe de concretarse a través del fomento de autonomía y competencia. En este sentido todos los agentes implicados en un proyecto de este tipo deben de respetar esta filosofia básica para lo que se formará a todo el personal.

Se seguirán los criterios del Sistema "Nursing Homes are living for" ("Las residencias son para vivir") para trabajar y garantizar todos estos aspectos: La INTIMIDAD: derecho de las personas a estar solas y a no ser molestadas, libres de intromisiones en sus asuntos; la DIGNIDAD: reconocimiento del valor intrínseco de la persona independientemente de cuales sean sus circunstancias, respetando su individualidad y sus necesidades, y dándole un trato respetuoso; La AUTONOMIA: posibilidad de actuar o de pensar de forma independiente, incluida la disposición a asumir ciertos niveles de riesgo calculado; la ELECCIÓN: posibilidad de elegir libremente entre un abanico de opciones diferentes; los DERECHOS: mantenimiento de todos los derechos inherentes a la condición de ciudadano; y la SATISFACCIÓN: realización de las aspiraciones personales y desarrollo de las capacidades de cada persona en todos los aspectos de la vida cotidiana.

\section{MODELO DE ATENCIÓN Y GESTIÓN}

El proceso de atención residencial tiene como objetivo la ejecución, siguiendo la planificación estratégica , de las actividades (ingreso, evaluación inicial, definición personalizada de necesidades, prestación de servicios, análisis de resultados, reevaluación programada y según necesidades, alta o traslado) que se llevan a cabo en los centros y unidades gerontológicas y residenciales para lograr la mejor calidad de vida de las personas mayores atendidas y sus familias, entendida esta como el mantenimiento y/o mejora de su autonomía, capacidad funcional, bienestar, satisfacción, integración y mantenimiento de relaciones sociales y familiares, así como garantizar y promover sus derechos consiguiendo satisfacer al máximo sus expectativas, con la máxima eficacia y eficiencia, buscando la máxima implicación y satisfacción de las personas y equipos profesionales y de las instituciones con quienes tenemos concertadas las plazas y siempre dentro de los presupuestos establecidos.

El proceso de atención residencial es el proceso clave asistencial que impacta directamente en el residente y su familia pero es complementado por otros de carácter estratégico y soporte como se puede ver en el MAPA de PROCESOS (Matia Fundazioa, 2004) y el proceso de 
atención residencial y de centro de dia se desarrolla siguiendo los siguientes pasos: ( figura 2).

\section{Influencia del entorno en la calidad de vida de las personas mayores}

Siguiendo los últimos avances en atención a las personas discapacitadas y dependientes, entendemos que la discapacidad o la necesidad de atención y apoyos no es fija ni dicotomizada, sino fluida, dinámica, continua y cambiante, dependiente de las limitaciones funcionales de la persona y de los apoyos disponibles en su entorno (Shalock y Verdugo, 2004). Hoy en día existen eviden- cias de que se pueden reducir las limitaciones funcionales, y por tanto la discapacidad, si se proporcionan servicios y apoyos centrados en la conducta adaptativa y en el estatus de los roles. Si la discapacidad es el resultado de la interacción de esta persona y su entorno, podemos tener una nueva visión de la discapacidad en la que la autodeterminación, la inclusión, la equidad y las fortalezas del sujeto desempeñan un papel fundamental.

Este paradigma se basa en ofrecer una vida con apoyos para lograr la mejor calidad de vida, fomentando la competencia personal y las conductas adaptativas a través de la formación y entrena-

Figura 2. Proceso de atención residencial

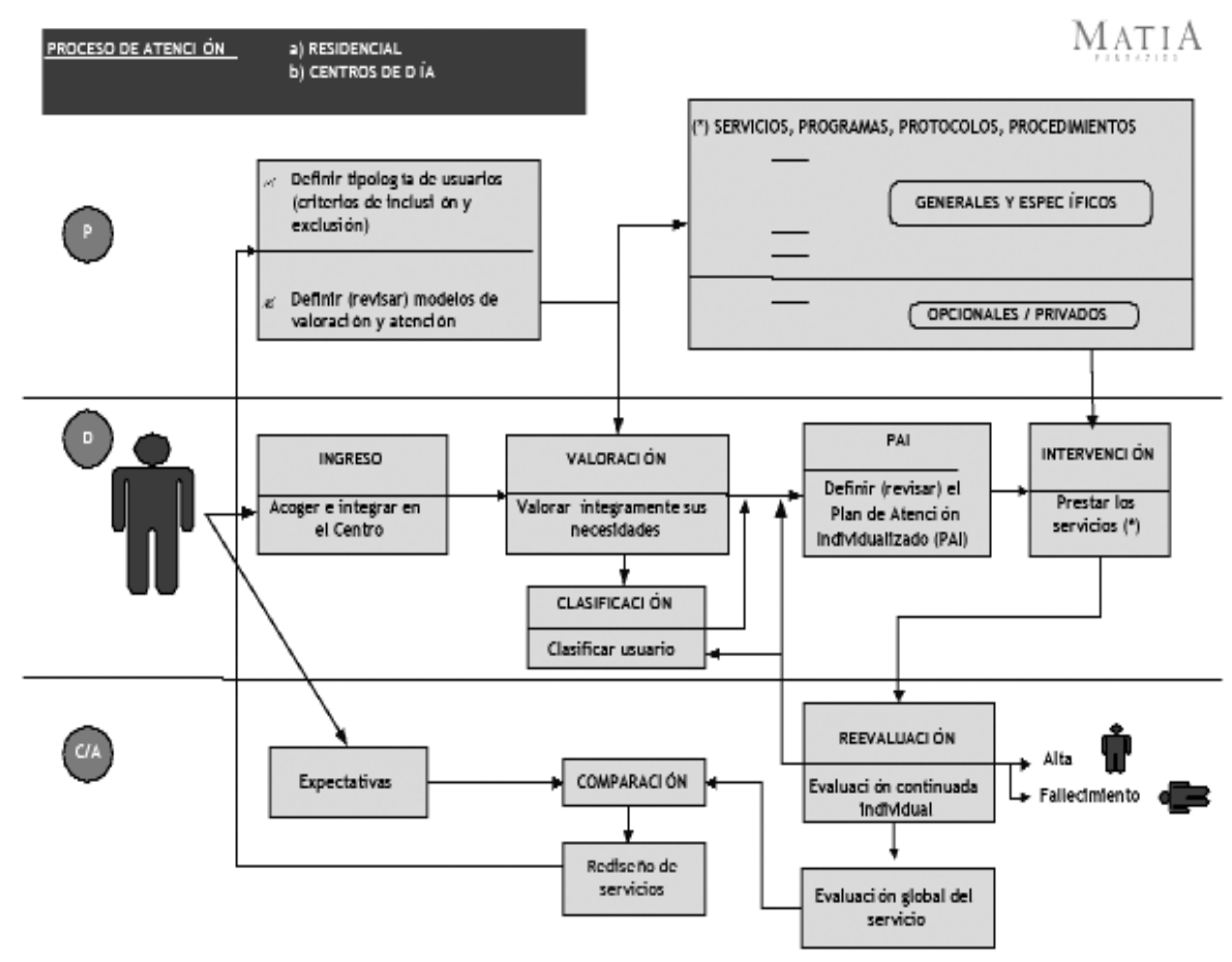


miento en habilidades, proporcionando apoyos, ayudas técnicas, oportunidades y capacidad de elección en entornos seguros, pero abiertos, libres y estimulantes para la implementación de estas competencias. ( figura 3)

La calidad de vida, entendida como el reflejo de las condiciones de vida deseadas por una persona en relación con sus necesidades vitales fundamentales, engloba aspectos como la satisfacción vital, el bienestar, la felicidad etc. (Fernández Ballesteros, 2000), comprende diferentes dimensiones y permite establecer indicadores y objetivos definidos para la intervención.

Los centros para personas mayores deben ofrecer recursos comunitarios y especializados para las personas mayores que necesitan ayuda para desenvolverse en la vida cotidiana, así como para sus familias. Estos recursos han de tener un carácter abierto, plural y multi- funcional, donde la calidad de vida es el objetivo principal. Entre las variables influyentes en la vida del centro destacan las características de las personas residentes, el modelo de atención y la capacidad del equipo profesional. En concreto, este equipo debe ofrecer una atención profesional y especializada de forma personalizada y cercana, en un entorno confortable, estimulante, orientador y protésico, adaptado a las necesidades de la persona y abierto a su familia.

Para las personas mayores en su domicilio y en recursos comunitarios los objetivos deberían ser similares, basándonos en la complementación al apoyo familiar ya existente.

En suma, desde este modelo el ajuste se logrará a partir de la interacción entre las capacidades de la persona y la adaptación del entorno y ambiente a esas capacidades.

Figura 3.
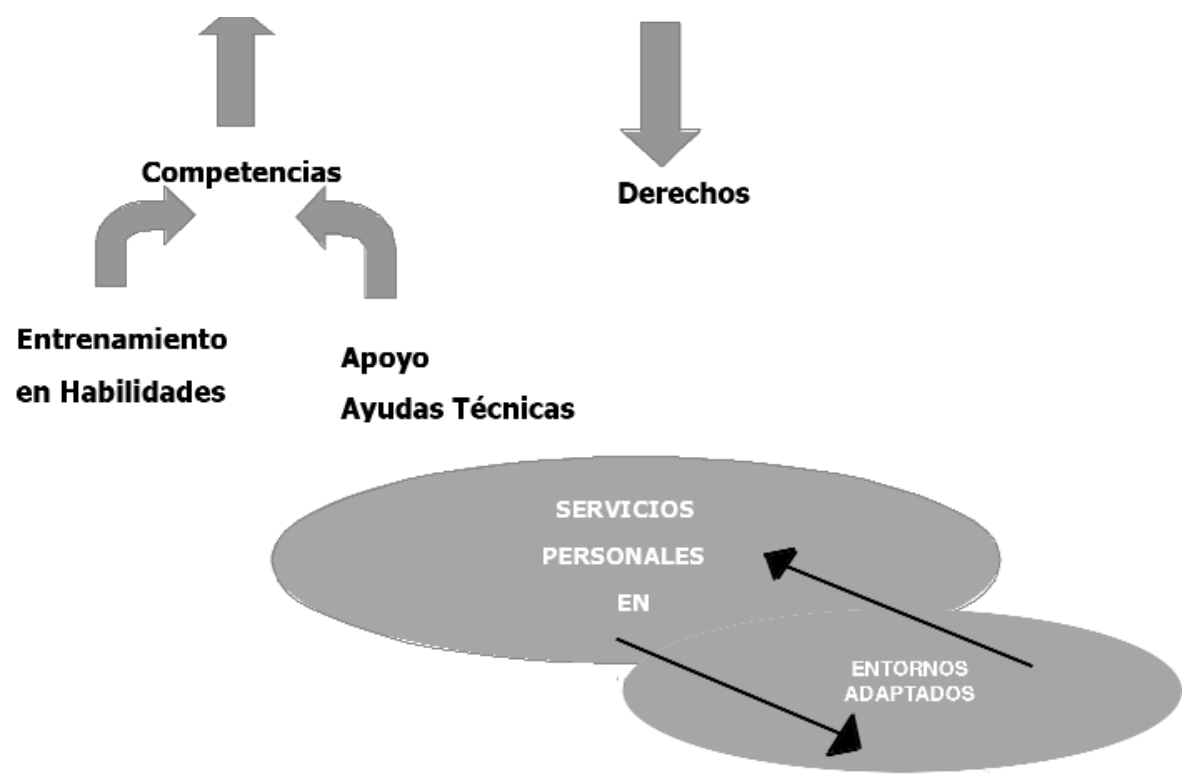


\section{CARÁCTER INTEGRAL DE INTERVENCIÓN}

Toda intervención gerontológica debe estar regida por ciertos principios. En primer lugar, debe centrarse en la normalización e integración (más que en la protección), en la defensa de los derechos y la creación de oportunidades, en la contextualización y la descategorización (Yanguas, Leturia, 1995)

En segundo lugar, debe partir de la complejidad de los procesos, problemas, situaciones que se dan en las personas con enfermedad crónica o discapacidad. En estas situaciones múltiples variables interrelacionadas se autodeterminan en sistemas complejos que conllevan influencias variadas según diferentes niveles de interacción. Ello hace necesario cualquier intervención, tanto de tipo profesional como personal, debe ser interdisciplinar y debe superar una perspectiva estrictamente individual (Yanguas, Leturia, 1995).

La base de este sistema de intervención se encuentra en una adecuada definición del proceso a partir de la acogida, valoraciôn, diagnóstico y establecimiento del Plan de Atención Individualizada.

La valoración, además de ser interdisciplinar, debe incluir desde el modelo psicosocial las principales áreas y objetivos (Leturia, Yanguas, Uriarte, 2001):

- Las capacidades mantenidas.

- Las discapacidades.

- La dependencia consecuente.

- Las necesidades.

- Las preferencias.
- Los valores y aspectos éticos relacionados.

El Plan General de Intervención (PGI) que aquí se expone consta de seis áreas diferenciadas como aparece recogido en la ilustración adjunta, que constituyen seis pilares de acción, que entendemos que tienen especial influencia en la calidad de vida de las personas mayores. Estas áreas son las siguientes: área de salud, relaciones sociales, cultura, integración y participación sociocomunitaria, comunidad, ambientes y el área de organización. (figura 4).

Los Programas de Intervención Psicosocial están procedimentados dentro del proceso de Gestión del Conocimiento, y son los siguientes: área cognitiva (Orientación en la Realidad, Entrenamiento en Memoria, Psicoestimulación Sensorial, Buenos Días, Lecto- escritura), área psicoafectiva (apoyo psicoafectivo, relajación, musicoterapia, apoyo a familias), salud física (gimnasia/ psicomotricidad, terapia ocupacional, prevención y promoción de la salud, laborterapia), relaciones sociales (apoyo social, voluntariado, formación a familias, día de familias), ocio y tiempo libre (aula de cultura, actividades y festividades), integración comunitaria (jornada de puertas abiertas, programa intergeneracional, colaboración en programas de radio, actividades en la comunidad), área de ambientes (Sera, Burnout).

La participación global en estos programas es de un $78,18 \%$.

El 75\% de los clientes son atendidos en programas de intervencion de carácter cognitivo, el 18 en programas de carácter psicoafectivo y emocional, el $48 \%$ en terapia ocupacional, y el 40 en programas de gimnasia y psicomotricidad. 
Figura 4. Plan General de Intervención

\section{DLAGNÚSTICO PSTCOSOCLAL Y SOCIOSANITARIO}

\begin{tabular}{|c|c|c|c|c|c|}
\hline $\begin{array}{l}\text { Kirra de } \\
\text { Salud }\end{array}$ & $\begin{array}{l}\text { Árca de Relaciones } \\
\text { Soctales }\end{array}$ & $\begin{array}{c}\text { Airea de } \\
\text { Odlo }\end{array}$ & Área Comvultarla & Área Ambleutal & Ára de Organizaclón \\
\hline 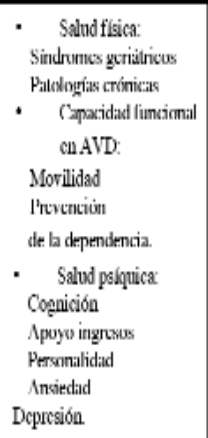 & 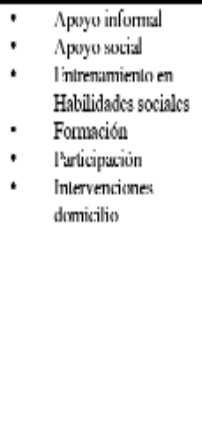 & 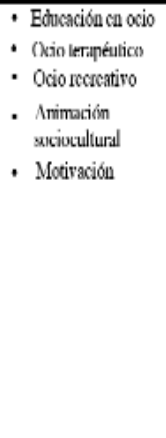 & 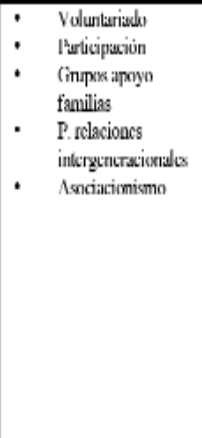 & 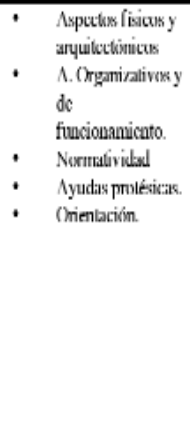 & 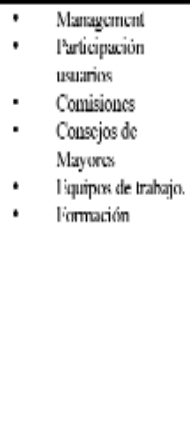 \\
\hline
\end{tabular}

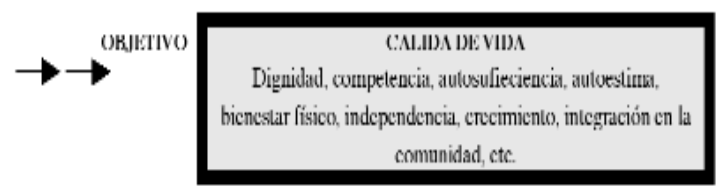

\section{INTERVENCION EN AREA COGNITIVA Y PSICOAFECTIVA}

Así como en los últimos años se ha avanzado bastante en la intervención y rehabilitación cognitiva otros problemas de carácter psicoafectivo y emocional como la depresión y la ansiedad en personas mayores han sido temas olvidados.

En nuestro caso le damos mucha importancia en si mismo como dimensión esencial en la calidad de vida y como factor coadyuvante en el resto de áreas de manera que en ocasiones tratamientos de carác- ter cognitivo o funcional solamente son eficaces si previamente se ha preparado a la persona desde objetivos y metodologías de carácter emocional y comportamental.

En el area cognitiva señalar la necesidad de desarrollar instrumentos de valoracion, estrategias y programas de intervención para las personas en fases avanzadas de la enfermedad o deterioro para evitar el nihilismo terapeútico imperante en general. Así se estan validando instrumentos de evaluación del deterioro cognitivo severo, así como procedimientos de intervención. 


\section{INTERVENCIÓN EN EL CUIDADO Y APOYO PERSONAL}

El primer objetivo será trabajar sobre la sobredependencia y examinar, mediante un análisis funcional, si la no ejecución de la conducta es debido a la discapacidad o a una falta de motivación para su realización. Algunos estudios y nuestra práctica avalan la eficacia de las estrategias conductuales en el entrenamiento y mantenimiento de comportamientos relacionados con la higiene, la deambulación, la alimentación, etc. (Leturia, Yanguas, 1999; Montorio, Izal, 1999).

El apoyo instrumental se centra en proporcionar a la persona y la familia ayudas para la realización de las actividades cotidianas de cuidado, limpieza, etc. La intervención con familiares ofreciéndoles formación y capacitando a las familias en esta área es un buen método no sólo para aumentar la calidad de vida de la persona mayor, sino para mejorar la implicación de las familias, al tener herramientas para poder realizar bien su labor de cuidado. Incluso con personas mayores residentes es factible formación de este tipo, para que sea puesta en marcha en el domicilio, cuando ocasionalmente están con la persona mayor, etc.

Estos programas incluyen la formación de familiares para que ayuden a las personas mayores dependientes a realizar actividades de vida diaria básica, instrumentales o complejas.

\section{INTERVENCIÓN EN EL CONTEXTO Y EL AMBIENTE}

Las características ambientales son muy importantes en los contextos pues afectan de manera muy relevante a la autonomía, al desempeño diario y, consecuentemente, a la satisfacción y calidad de vida de las personas. Estas características ambientales no se limitan a sólo a aspectos físicos. También son muy relevantes las características organizativas, las características de los residentes y del personal, las relaciones interpersonales o el clima social (Fernández Ballesteros, 1996).

Para poder evaluar el ambiente y lograr un diseño ambiental que nos permita promover los objetivos conocidos de autonomía, satisfacción y calidad de vida se debe operacionalizar estas dimensiones ambientales en aspectos concretos como la intimidad, la integración social, el control y posibilidad de elección, etc. (Regnier, 1993).

Entre los instrumentos de evaluación ambiental de residencias destaca el SERA Sistema de Evaluación de Residencias de Ancianos de Fernández Ballesteros (1997). Este instrumento distingue las siguientes áreas generales: a) características físicas (accesibilidad la comunidad, las características de seguridad, las ayudas para la orientación, el confort físico etc.); b) aspectos organizativos (la intimidad, el control por los residentes, la organización, la elección organizativa, la disponibilidad de servicios etc.) c) características de residentes y de personal, (el nivel de habilidades funcionales de los residentes, su nivel de actividad, su integración en la comunidad, la utilización de servicios etc.) El clima social que comprende la calidad de las relaciones interpersonales, las posibilidades de desarrollo personal, las tendencias de estabilidad o cambio en el sistema, etc.

El diseño ambiental permitirá a través de la modificación del ambiente influir en problemas de comportamiento como la deambulación, los riesgos de caídas, o los déficits sensoriales que pueden comprometer la autonomía, para lo que se proporcionan las prótesis adecuadas como bastones, sillas de ruedas, eliminación de barre- 
ras arquitectónicas. Colocando correctamente el mobiliario podemos, por ejemplo, favorecer también el contacto y las relaciones sociales (Fdez Ballesteros et al. 1992) Una mejora de los estimulos ambientales hará que la persona mayor responda más fácilmente y de manera orientada en el tiempo y espacio (figura 5).

\section{INTERVENCIÓN EN APOYO SOCIAL Y FAMILIAR}

Desde este modelo la intervención con las personas no se limita solamente a los residentes, mayores y/o discapacitados y usuarios de centro de día sino también a sus familiares entendiendo que siguen constituyendo un sistema familiar y que por tanto debemos seguir atendiendo a las necesidades del sistema en su conjunto, o cuando menos a los cuidadores principales. Los programas de apoyo social y de intervención con familias son determinantes para las personas dependientes y sus familiares así como para el personal.

\section{INTERVENCIÓN EN EL ÁMBITO COMUNITARIO}

Además del análisis comunitario, la realización del Mapa comunitario" en el que se analizarán todas las relaciones con los recursos ( educativos, sociales,

\section{Figura 5. ICOF Comparativa entre Centros}

ICOF

ICOF

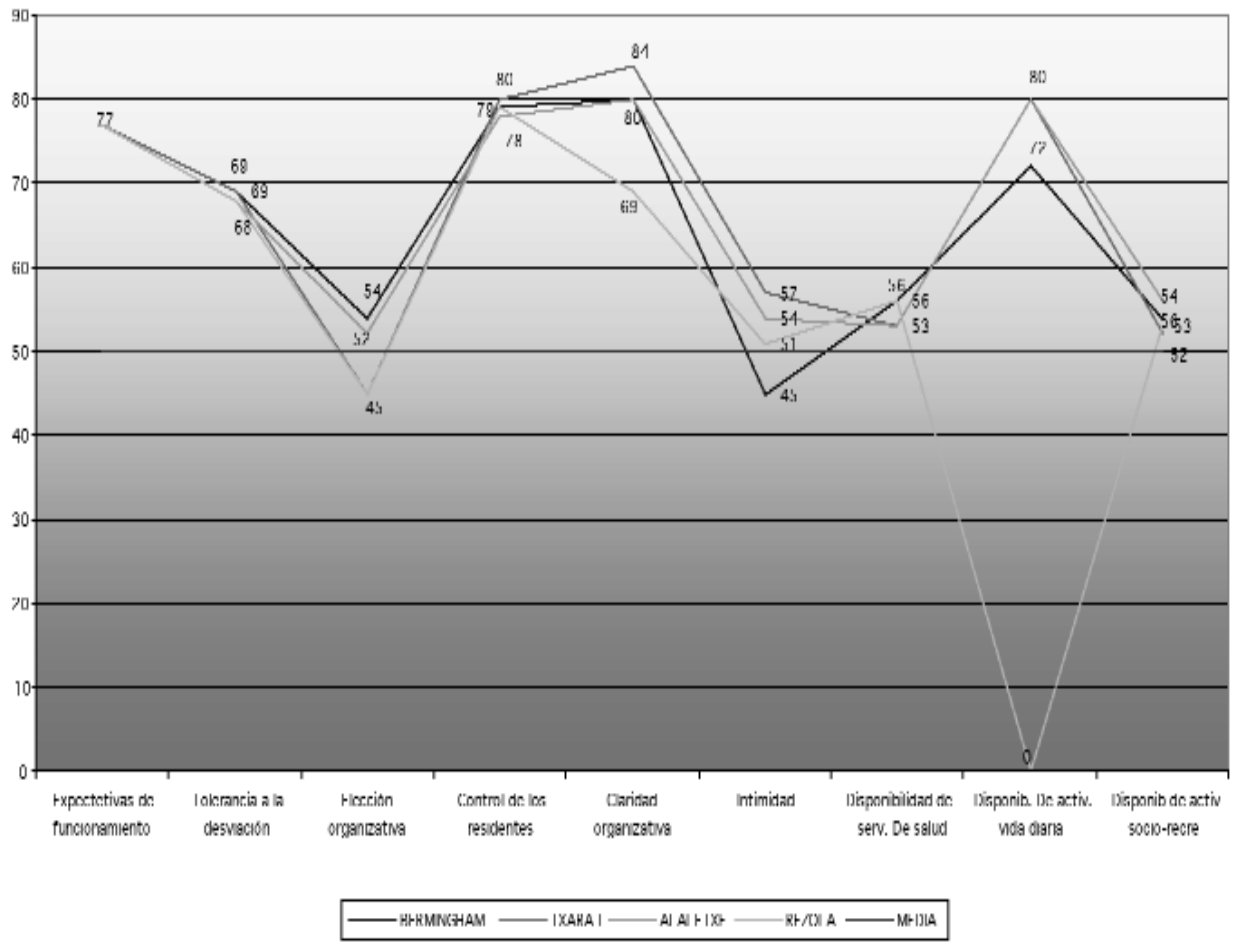


sanitarios, "sectoriales" etc. y asociaciones del entorno, se trabajará con ellos, se les informará de nuestros objetivos, se les invitara a un Día de Puertas abiertas, se analizarán otros potenciales partenaires de proyectos etc. Se planifica y realiza una intervención con las asociaciones y recursos del entorno, programas de relaciones intergeneracionales y programas de fomento de voluntariado y la generación de las Escuelas de la experiencia y el fomento de asociacionismo, creación de la Asociación MUGABE de asociaciones surgidas desde los programas formativos etc.

\section{INTERVENCION EN ASPECTOS ORGANIZACIONALES}

Además de la importancia del liderazgo desde modelos organizativos y culturales avanzados en gestión, la gestión por competencias de las personas cuidadoras y técnicos, es importante señalar la capacitación de las mismas, el entrenamiento y el apoyo para atender a colectivos que presentan cada vez mayor complejidad y por tanto mayores niveles de formación, de gestión de los riesgos psicosociales y emocionales, gestión de equipos interdisciplinares etc.

\section{RESULTADOS:}

Como resultados de la implantación de este modelo de atención psicosocial y de gestión basado en procesos y mejora continua según el modelo EFQM podemos destacar que el área psicosocial en Matia Fundazioa ha sufrido una evolución importante en los últimos años, a partir de la implantación del nuevo modelo de Atención e Intervención Psicosocial de manera integrada a los modelos de gestión más avanzados y a un enfoque abierto a la comunidad y orientado al cliente.

\section{Implantación de Programas de} intervención psicosocial. En el Área cognitiva el número de programas ha sufrido un gran incremento con el tiempo, pasando de 10 en los años 1998/99 a 35 en el 2005; En el Área psicoafectiva también se ha producido un gran cambio, pasándose de 2 programas en el 98 a 27 en el 2005; En el Área de salud física pasamos de 9 programas en el 98 a 33 en el 2005; En el Área de ocio y tiempo libre sin cambios a lo largo de estos años; En el Área de relaciones sociales la intervención comenzó en el 2002; En el Área de ambientes en el 2005 se realiza el SERA en cuatro de los centros de Matia Fundazioa; La media de participación en programas es muy alta en todos los centros (78.18\% en el 2005).

\section{Mejora de la satisfacción directa} del cliente tanto usuario como familia que se realiza a través de las encuestas que revelan el incremento en satisfacción de los usuarios tanto en general como con el funcionamiento y la organización y diferentes aspectos de la atención, la personalización de la misma, etc. (Figura 6) (figura 7). (Figura 8 y 9).

3. Integración de modelo de atención psicosocial y sociosanitario y el modelo de gestión y desarrollo de una cultura organizacional orientada a las personas y sus familias.

4. Adaptación progresiva de la organización a un nivel de dependencia cada vez mayor y de caracter importantemente psicogeriátrico.

5. Importante avance en la capacitación de las personas y en el desarrollo de un modelo de gestión por competencias. 
Figura 6. Satisfacción global familias y usuarios

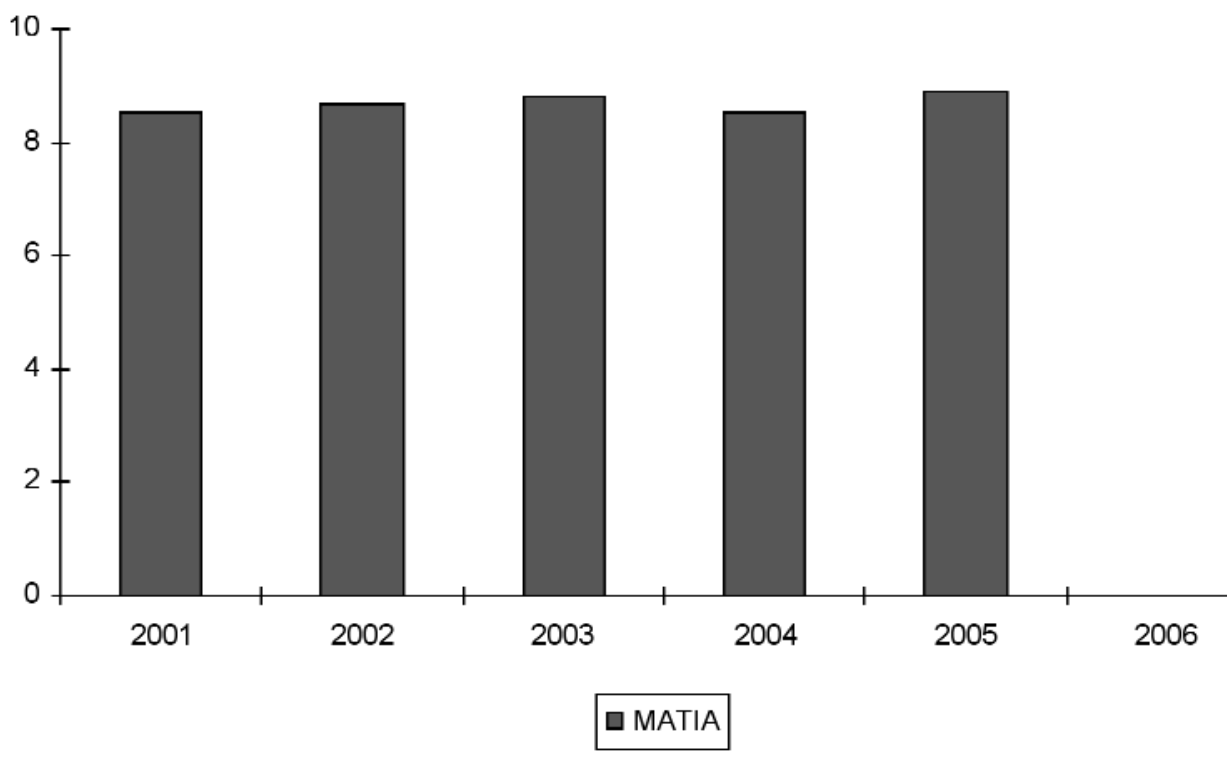

Figura 7. Satisfacción comunidad y voluntarios MEDIA SATISFACCIÓN COMUNIDAD Y VOLUNTARIOS CENTROS MATIA

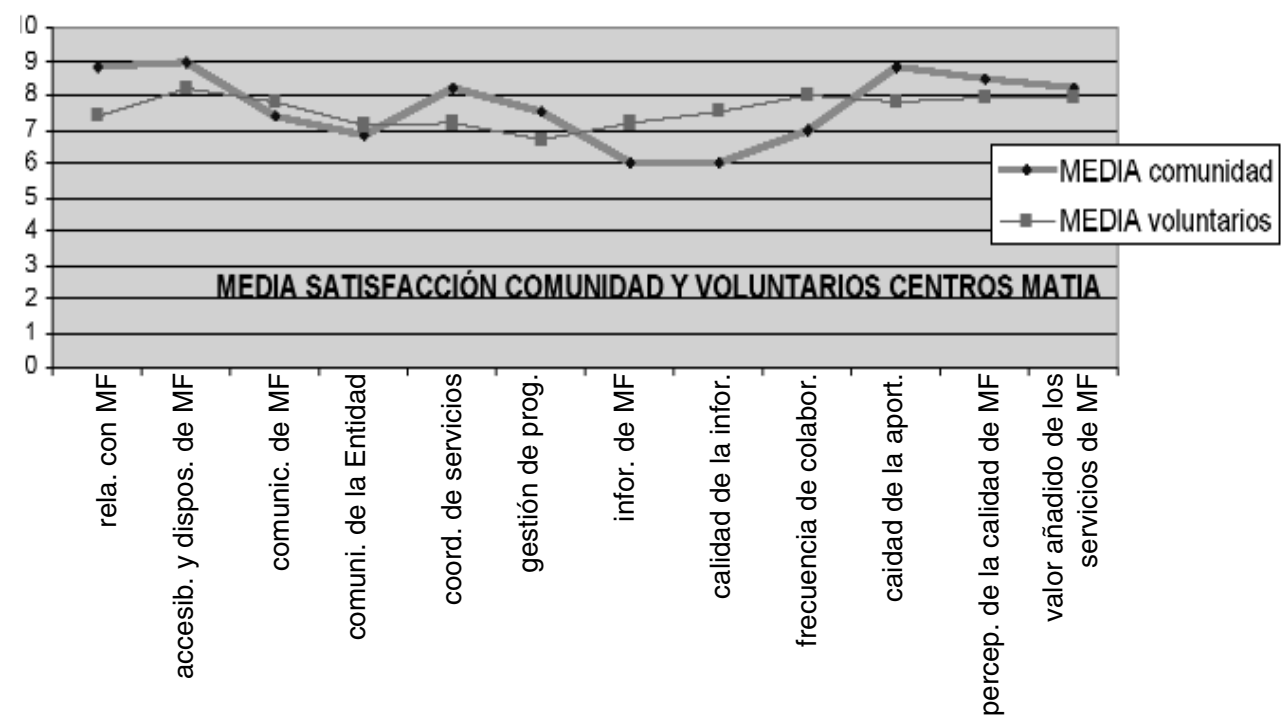


Figura 8. Satisfacción con el funcionamiento y la organización

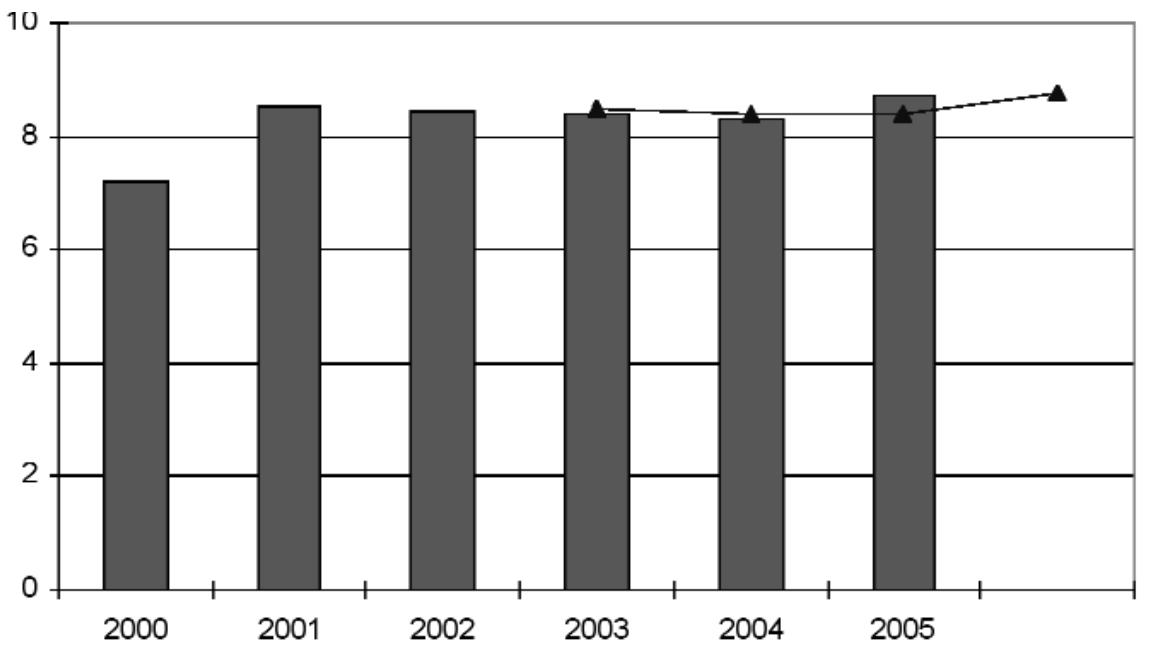

Figura 9. Satisfacción con la atención necesidades

$\square 2000 \square 2001 \square 2002 \square 2003 \square 2004 \square 2005$

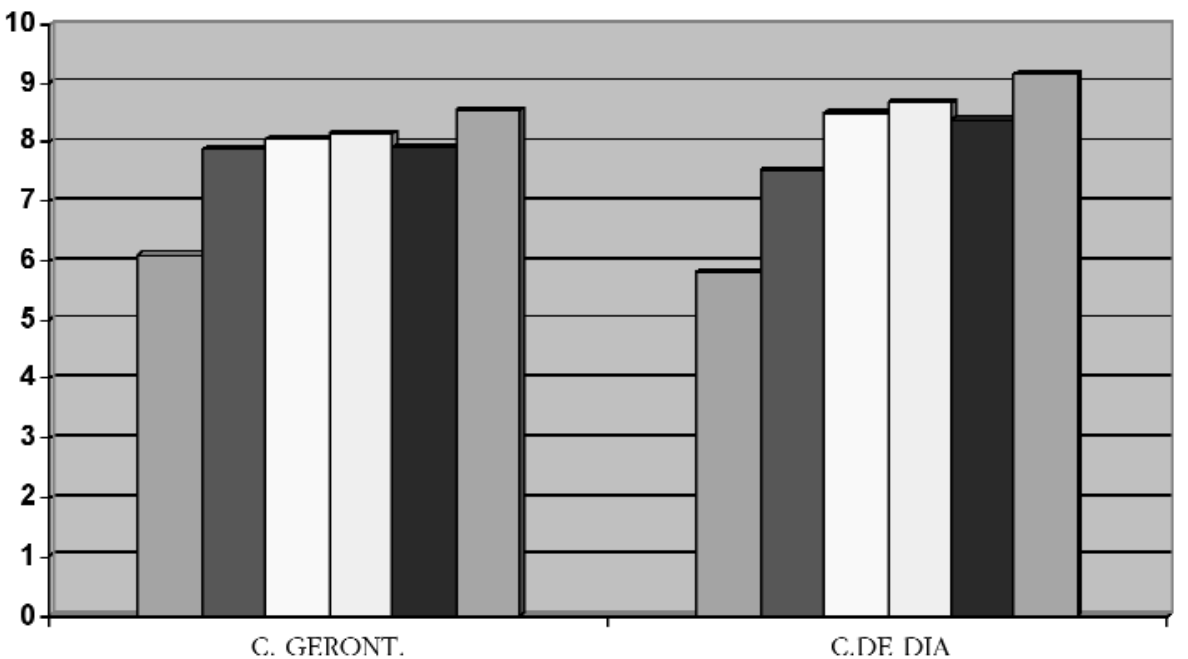

6. No obstante se presentan nuevos retos como el avance en el desarrollo de valoraciones más específicas, adaptadas a avanzados niveles de deterioro, un análisis más exhaus- tivo sobre la eficacia de las diferentes intervenciones, la sistematización de las mismas, la gestión adecuada de las personas, la gestión de los riesgos en la atención, etc. 
Figura 10. Satisfacción con trato y actitud

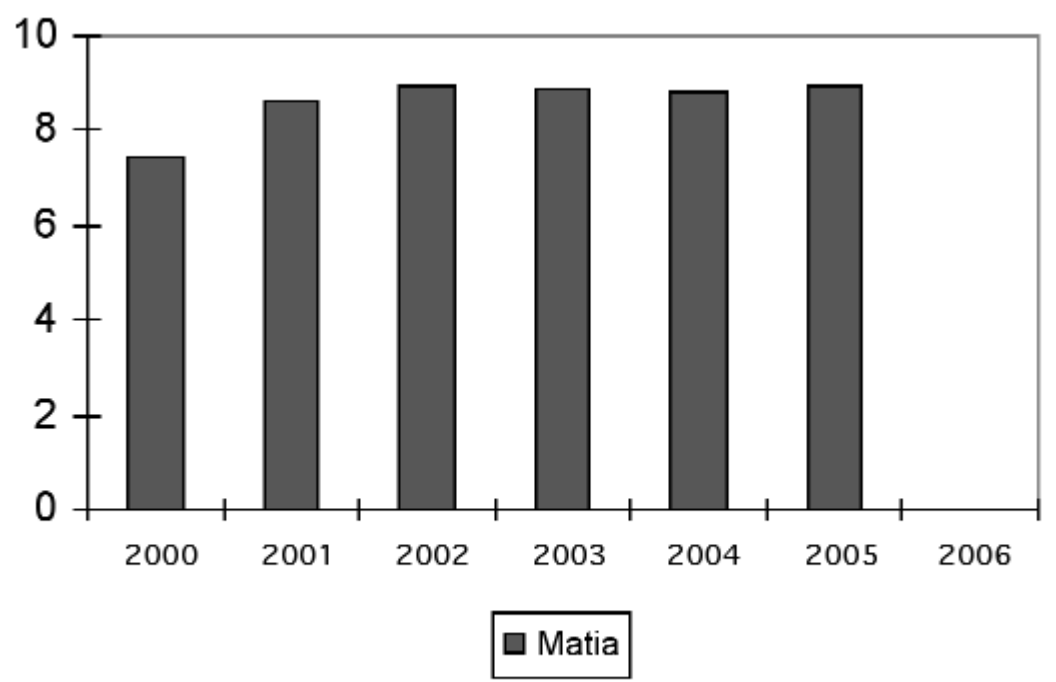

\section{BIBLIOGRAFÍA}

Baltes P.B. y Baltes, M.M. (1990). Successful aging: perspectives from the behavioral sciencies. Cambridge University Press.

Baltes, M.M. y Wener, W. (1990). Dependencia en los ancianos. En Carstensen y Edelstein (1990): Gerontología clínica: intervención psicológica y social. Martinez Roca, Madrid.

Fernández Ballesteros, R. (1997). Sistema de evaluación de residencias de ancianos (SERA). IMSERSO. Madrid.

Fernández Ballesteros, R. (1996). Evaluación e Intervención psicológica en la vejez. Madrid. 1996. Martínez Roca.

Leturia F.J. y Yanguas, J.J., (1998). Intervención psicosocial en gerontologia: manual práctico.Caritas Española, Madrid.

Montorio I. e Izal, M. (1999). Gerontología conductual: intervención y ámbitos de aplicación. Síntesis. Madrid.
Leturia, F.J., Yanguas, J.J., Uriarte, A. y otros (2001). La valoración de las personas mayores: evaluar para conocer, conocer para intervenir. Cáritas Española. Madrid.

Montorio, I. (1995). La atención psicológica. En P. Rodríguez ( de): Residencias para personas mayores. Manual de Orientación. S. G., Madrid.

Montorio Cerrato, I., y Losada Baltor, A. (2004). Una visión psicosocial de la dependencia desafiando la perspectiva tradicional. Perfiles y tendencias octubre 2004. Madrid: IMSERSO.

Moos, R.H. (1979). Evaluating Educational Environments: Procedures, Measures, Findings, and Policy Implications. San Francisco, JosseyBass.

Yanguas, J.J. y Leturia, F.J. (1995a y 1995b). Intervención con familias. En AA.VV. Residencias para personas mayores. Sociedad Española de Geriatria y Gerontologia, Barcelona. 